Konstanzer Online-Publikations-System (KOPS)

URL: http://www.ub.uni-konstanz.de/kops/volltexte/2008/470/

URN: http://nbn-resolving.de/urn:nbn:de:bsz:352-opus-47010

Fakultät für Biologie, Lebrstubl für Phytopatbologie

der Universität Konstanz

\title{
Einsatz von $V$. lecanii als biologisches Schädlingsbekämpfungsmittel gegen den Bohnenrostpilz \\ $U$. appendiculatus var. appendiculatus im Feld und im Gewächshaus
}

\author{
Von \\ G. C. Grabski und K. Mendgen
}

Mit 3 Abbildungen

Eingegangen am 21. Dezember, 1984

\begin{abstract}
The use of Verticillium lecanii as a biological control agent against the bean rust fungus Uromyces appendiculatus var. appendiculatis in the field and in the glasshouse
\end{abstract}

The deuteromycete $V$. lecanii parasites uredo- and teliospores of the bean-rust-fungus $U$. appendiculatus var. appendiculatus. We investigated the conditions for the use of the hyperparasite as biological control agent in the field and in glasshouses.

The growth rate of the hyperparasite was $0,3 \mathrm{~cm}$ per day $2 t 25^{\circ} \mathrm{C}$. Under suitable conditions in the lab $\left(25^{\circ} \mathrm{C}, 100 \%\right.$ r. h. $)$ it took about 20 days to invade $100 \%$ of uredospores and $65 \%$ of teliospores.

We failed to prevent the spread of bean-rust-fungus spores in the field, but we succeeded in the glasshouse by $68 \%$, compared to the untreated controls, using the hyperparasite $V$ : lecanii as biological control agent.

\section{Zusammenfassung}

Der Deuteromycet $V$. lecanii befällt die Uredo- und Teleutosporen des Bohnenrostpilzes $U$. appendiculatus var, appendiculatus.

Es wurde untersucht, unter welchen Bedingungen und mit welchem Erfolg der hyperparasitäre Pilz im Freiland und im Gewächshaus zur biologischen Bekämpfung des Bohnenrostpilzes eingesetzt werden kann.

Die Wachstumsrate des Hyperparasiten betrug bei $25^{\circ} \mathrm{C} 0,3 \mathrm{~cm}$ pro Tag. Unter optimalen Bedingungen im Labor $\left(25^{\circ} \mathrm{C}, 100 \%\right.$ r. L.) wurden Uredosporen nach 20 Tagen zu $100 \%$ und Teleurosporen nach 20 Tagen zu $65 \%$ infiziert. 
Versuche, die Ausbreitung des Bohnenrostpilzes im Feld durch den Einsatz von V. lecanii zu verhindern, scheiterten. Im Gewächshaus dagegen konnte die Verbreitung der Rostsporen zu $68 \%$ unterbunden werden, verglichen mit der Ausbreitung in der unbehandelten Kontrollparzelle.

Im Rahmen des "Integrierten Pflanzenschutzes“ ist es von zunehmender Bedeutung, die Biologie natürlicher Antagonisten von Schaderregern aufzuklären und zu prüfen, inwieweit sich diese „nützlichen" Organismen als Hilfsmittel im Pflanzenschutz einsetzen lassen.

Lebensbedingungen, wie z. B. Temperatur- und Luftfeuchteansprüche, sowie Möglichkeiten zum künstlichen Masseneinsatz und zur Lagerung von Infektionsmaterial müssen ebenso untersucht werden, wie die Spezifität und Effektivität des "nützlichen“ Organismus.

Bereits seit 1936 wird der Hyperparasit V. lecanii aus Bodenproben, zusammen mit Rostpilzsporen, oder von rostpilzinfiziertem Pflanzenmaterial isoliert und beschrieben (DOMSCH 1960, Finamagan und SCARBorough 1974, Gams 1971, Hassebrauk 1936, Hering 1965, v. Schroeder und Hassebraur 1957). Die Abhängigkeit des Befalls von Uredolagern des Getreidegelbrostes Puccinia striiformis von der Lichtstärke, Temperatur und Luftfeuchtigkeit wurde von Mendgen untersucht (MENDGEN 1979 a, b). Licht- und Elektronenmikroskopie dokumentieren den Zerfall von Uredo- und Teleutosporenlagern der Rostpilze (GrabSKI eingereicht, HäNSSLER et al. 1981, MENDGEN 1981). Die vorliegende Arbeit berichtet von unseren Versuchen, die Biologie des Hyperparasiten weiter aufzuklären, ihn in einem Masseneinsatz im Feld und Gewächshaus auszubringen und seine Wirkung gegen die Ausbreitung des Bohnenrostpilzes $U$. appendiculatus var. appendiculatus zu prüfen.

\section{Material und Methoden}

\section{Vermehrung von $V$. lecanii}

In der vorliegenden Arbeit wurde mit dem V. lecanii Isolat VK 1 gearbeitet, das 1977 in Göttingen von Gelbrost isoliert wurde. Zur Gewinnung großer Volumina von Verticilliumsporen in Suspension wurden $30 \mathrm{~g} / 1$ Biomalz in aqua dest. gelöst und $20 \mathrm{Min}$. bei $120^{\circ} \mathrm{C}$ autoklaviert. Myzel des Hyperparasiten wurde steril in die Kolben überführt und bei $25^{\circ} \mathrm{C}$ und $200 \mathrm{UpM}$ in einem Schürtler (Fa. Brunswick) inkubiert. Nach 10 Tagen wurden Myzel und Sporen durch Filtration durch eine Zellstoffgaze getrenot. Die Sporen wurden in einer Sigma $2 \mathrm{KD}$ Minifuge bei $4500 \mathrm{UpM}$ und $4{ }^{\circ} \mathrm{C}$ für $10 \mathrm{Min}$. oder in einer Sorvall RC 5B Superspeed Zentrifuge bei $4000 \mathrm{~g}$ und $4^{\circ} \mathrm{C}$ für $15 \mathrm{Min}$. gewaschen. Die Sporensuspension wurde auf eine Konzentration von $10^{8}$ Sporen $/ \mathrm{ml}$ eingestellt und sogleich verwendet.

\section{Untersuchungen zum temperaturunabhängigen $W$ achstum von $V$. lecanii, mit/ohne Lichteinwirkung}

Von einer 16 Tage alten $V$. lecanii Kultur auf Malzagar wurden 21 Scheiben von $0,5 \mathrm{~cm}$ Durchmesser ausgestanzt und auf je 1 frische Agarplatte überführ. 1 beimpfte Agarplatte diente als neue Stammkultur, die anderen Platten wurden bei verschiedenen Temperaturen bebrütet. 10 Platten wurden mit Aluminiumfolie abgedeckt, die anderen 10 Platten wurden mit 10000 Lux aus Quecksilberdampflampen beleuchtet. Die Auswertung erfolgte nach 16 Tagen durch Ausmessen des Koloniedurchmessers. Danach wurde die nächste Temperatur gewähit und Agarplatten wie oben beschrieben beimpft. Wachstum von $V$. lecanii wurde bei $4,10,15,20,25$ und $30^{\circ} \mathrm{C}$ getestet. 


\section{Zeitabhängigkeit der Infektion}

Uredo- und Teleutosporen eines Rostpilzisolates von der Insel Reichenau (RBR), freundlicherweise von Herm Dr. R. Golo zur Verfügung gestellt, wurden mit FC 40 vermischt und mit einem Pinsel auf einen "Sartorius"-Filterpapierstreifen aufgestrichen. Das Filterpapier wurde auf eine Wasseragarplatte (1,5\% Agar in Aqua dest., autoklaviert bei $120^{\circ} \mathrm{C}, 20 \mathrm{Min}$.) aufgebracht und mir

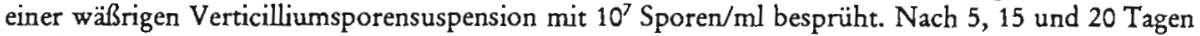
wurden Proben entnommen und im Zeiss-Lichtmikroskop untersucht. Es wurden jeweils ca. 500 Sporen ausgewertet und der Prozentsatz der infizierten Sporen ermittelt.

\section{Freilandversuche}

Im Sommer 1981 und 1982 wurden Freilandversuche im botanischen Garten der Universität Konstanz in Egg durchgeführt. Anfang Mai wurden Sarnen der Stangenbohne "Neckarkönigin" ausgelegt und mit wenig Torf bedeckt. Insgesamt wurden 54 Stangen gesteckt und $z$ war so, daß sich 6 Reihen à 9 Stangen ergaben. Hatten die ausgekeimten Bohnen eine Höhe von ca. $1,5 \mathrm{~m}$ erreicht, wurden sie mit einer Suspension mit $0,33 \mathrm{mg} / \mathrm{ml}$ Uredosporen des RBR Isolates besprüht, der 0,05\% v/v Tween 20 und $0,2 \mathrm{mg} / \mathrm{ml}$ Talkum zugesetzt wurde. Inokuliert wurden nur die Reihen 2 und 5. Benachbarte Reihen wurden von einer Plastikplane vor Inokulation geschützt. Erste Rostpusteln traten nach etwa 10 Tagen auf. Zu diesem Zeitpunkt wurde die Reihe 5 mit einer wäßrigen Verticilliumsporensuspension mit $10^{8} \mathrm{Sporen} / \mathrm{ml}$ inokuliert.

Wachstum des hyperparasitären Pilzes und Ausbreitung des Bohnenrostpilzes im Fịeld wurde täglich geprüft. Wiederholungen von Inokulationen mit Sporen des Hyperparasiten erfolgten im Abstand von ca. 10 Tagen. Eine Kontrolle des Vorbandenseins von V. lecanii im Feld wurde durchgeführ, indem ein Teil der Versuchsreihe 5 mit einer Plastikplane abgedeckt wurde, wodurch ein Treibhauseffekt bewirkt wurde.

Luftdruck, -temperatur und -feuchte wurden in unmittelbarer Nähe mittels eines Thermohygrographen gemessen und aufgezeichnet.

\section{Gewächshausversuche}

Im Sommer 1982 und 1983 wurden. Versuche in Gewächshaus der Universität Konstanz durchgeführt, das mit Klappenbelüftung und Mattenbefeuchtung betrieben wurde. Dabei wurde die Verhinderung der Ausbreitung von Bohnenrost in Gewächshauskulturen geprüft, indem Buschboh-

Tabelle 1

Versuchsanlageplan/Gewächshaus

\begin{tabular}{|c|c|c|c|c|c|c|c|c|c|c|}
\hline Reibe & $\because$ & 1 & 2 & 3 & 4 & 5 & 6 & 7 & 8 & 9 \\
\hline \multirow[t]{4}{*}{${ }_{n} \mathrm{R}^{\alpha}$} & $\mathbf{a}$ & 0 & $\mathrm{R}$ & 0 & $R \rightarrow ?$ & 0 & $\mathrm{R}$ & 0 & $\mathrm{R} \rightarrow$ ? & 0 \\
\hline & $b$ & $0 ? \leftarrow$ & $\mathrm{R}$ & 0 & $\mathrm{R}$ & $0 ? \leftarrow$ & $\mathrm{R}$ & 0 & $\mathrm{R}$ & 0 \\
\hline & $c$ & 0 & $\mathrm{R}$ & $0 ? \leftarrow$ & $R$ & 0 & $\mathrm{R}$ & $0 ? \leftarrow$ & $\mathrm{R}$ & 0 \\
\hline & $\mathrm{d}$ & 0 & $\mathrm{R} \rightarrow$ ? & 0 & $\mathrm{R}$ & 0 & $R \rightarrow ?$ & 0 & $\mathrm{R}$ & 0 \\
\hline
\end{tabular}

Plane

$\begin{array}{rllllllllll}{ }_{n} \mathrm{~V}^{\alpha} & \mathrm{a} & \mathrm{o} & \mathrm{V} & \mathrm{V} & \mathrm{V} \rightarrow ? & 0 & \mathrm{~V} & 0 & \mathrm{~V}\end{array} \rightarrow ? 0$

$0=$ unbehandelte Pflanzen

$R=$ Pflanzen mit Bohnenrost infiziert

$V=$ Pflanzen mit Bohnenrost infiziert und mit $V$. lecanii besprüht 
nen der Sorte "Fori“ in 9 Reihen à 8 Plastiktöpfe gesetzt wurden. Das Versuchsfeld wurde mit einer Plastikplane so aufgeteilt, daß sich zwei Hälften mit 9 Reihen à 4 Töpfen ergaben. In der Hälfte " $\mathrm{R}^{*}$ wurde die Ausbreitung des Bohnenrostes ohne $V$. lecanii, in der Hälfte „V $\mathrm{V}^{\alpha}$ dagegen wurde der Einfluß des Hyperparasiten auf die Rostpilzverbreitung geprüt (s. Tab. 1). Die Anzucht fand unter überwiegend natürlichen Lichtbedingungen statt, im späteren Herbst wurde zusätzlich mit Quecksilberdampflampen beleuchtet, so daß im gesamten Versuchszeitraum eine Lichtphase von $14 \mathrm{Std}$. gegeben war. Die Temperaturen sanken tagsüber nicht unter $20^{\circ} \mathrm{C}$, nachts nicht unter $15^{\circ} \mathrm{C}$. Die Infektion mit Rostsporen erfolgte nacb ca. 30 Tagen unter Verwendung eines preßluftbetriebenen Lacksprïhgerätes. Inokuliert wurden die Bohnen der Reiben 2, 4, 6 und 8. Die Inokulation wurde außerhalb der Gewächshauskammer durchgeführt, um unbeabsichtigte Infektionen in den Kontrollen zu vermeiden. Rostpusteln wurden nach ca. 10 Tagen gebildet. Zu diesem Zeitpunkt erfolgte die erste Spritzung mit einer $V$. lecanii Sporensuspension mit $10^{8} \mathrm{Sporen} / \mathrm{ml}$ in der Versuchshälfte ${ }_{n} V^{\alpha}$, in den Reihen 2, 4, 6 und 8. Gleichzeitig wurde die rel. Luftfeuchte auf über $90 \%$ erhöht, indem bei geschlossenen Lüftungsklappen ständig Wasser in die Kammer gepumpt wurde. Die Inokulation von Verticilliumsporen wurde alle 3 Tage wiederholt, bis Myzel des Hyperparasiten makroskopisch sichtbar wurde. Die Auswertung der Versuche erfolgte in der Regel 2 Monate nach Versuchsbeginn. Ausgewertet wurden die Anzahl Blätter/Topf, die Anzahl Rostpusteln pro Blatt, die Anzahl Pflanzen/ Topf, die Anzahl Früchte/Topf und das Gewicht des Ertrages/Topf.

\section{Ergebnisse}

\section{Untersuchungen zum temperaturabhängigen Wachstum von $V$. lecanü mit/ohne Lichteinwirkung}

Es wurde festgestellt, daß zwischen 4 und $15^{\circ} \mathrm{C}$ eine annähernd lineare Wachstumsgeschwindigkeitszunahme vorliegt (Korrelationskoeffizient $\mathrm{r}=$ 0,999). Das Temperaturoptimum wurde mit $25^{\circ} \mathrm{C}$ ermittelt. Bei $30^{\circ} \mathrm{C}$ verringerte sich die Wachstumsrate wieder: Unter Lichteinwirkung auf $48 \%$, ohne Lichteinwirkung auf 72,5\%, bezogen auf die maximale Wachstumsrate (Abb. 2).

\section{Zeitabhängigkeit des Infektionsverlaufes}

Die Infektion von Uredo- und Teleutosporen wurde über einen Zeitraum von 20 Tagen beobachtet. Nach 5, 15 und 20 Tagen wurden jeweils ca. 500 Sporen ausgewertet und das Ausmaß des Befalls durch den Hyperparasiten festgestellt. Uredosporen wurden nach 5 Tagen zu $14 \%$ und nach 15 Tagen zu $99 \%$ infiziert. Teleutosporen wurden nach 5 Tagen nicht, und nach 15 Tagen zu $5,7 \%$ infiziert. Nach 20 Tagen sind $100 \%$ der Uredo- und $65 \%$ der Teleutosporen des Bohnenrostpilzes vom Hyperparasiten befallen. Abb. 1 zeigt die graphische Darstellung des Infektionsverlaufs, der durch den stark verzögerten Befall der Teleutosporen gekennzeichnet ist.

\section{Biologische Bekämpfung im Freiland}

Ausgewertet wurde der Befall mit Rostpusteln auf Blättern der unbehandelten Bohnenreihen. Eine gleichmäßige Ausbreitung des Bohnenrostpilzes über das gesamte Versuchsfeld wurde festgestellt. Mit einer Lupe konnte Myzel des Hyperparasiten in der behandelten Reihe 5 nachgewiesen werden, makroskopisch sichtbar wurde es jedoch erst durch das Bedecken eines Teils der Versuchsreihe mit einer Plastikplane, wodurch das Wachstum des Hyperparasiten wegen der künstlichen Erhöhung der rel. Lufffeuchtigkeit begünstigt wurde. 


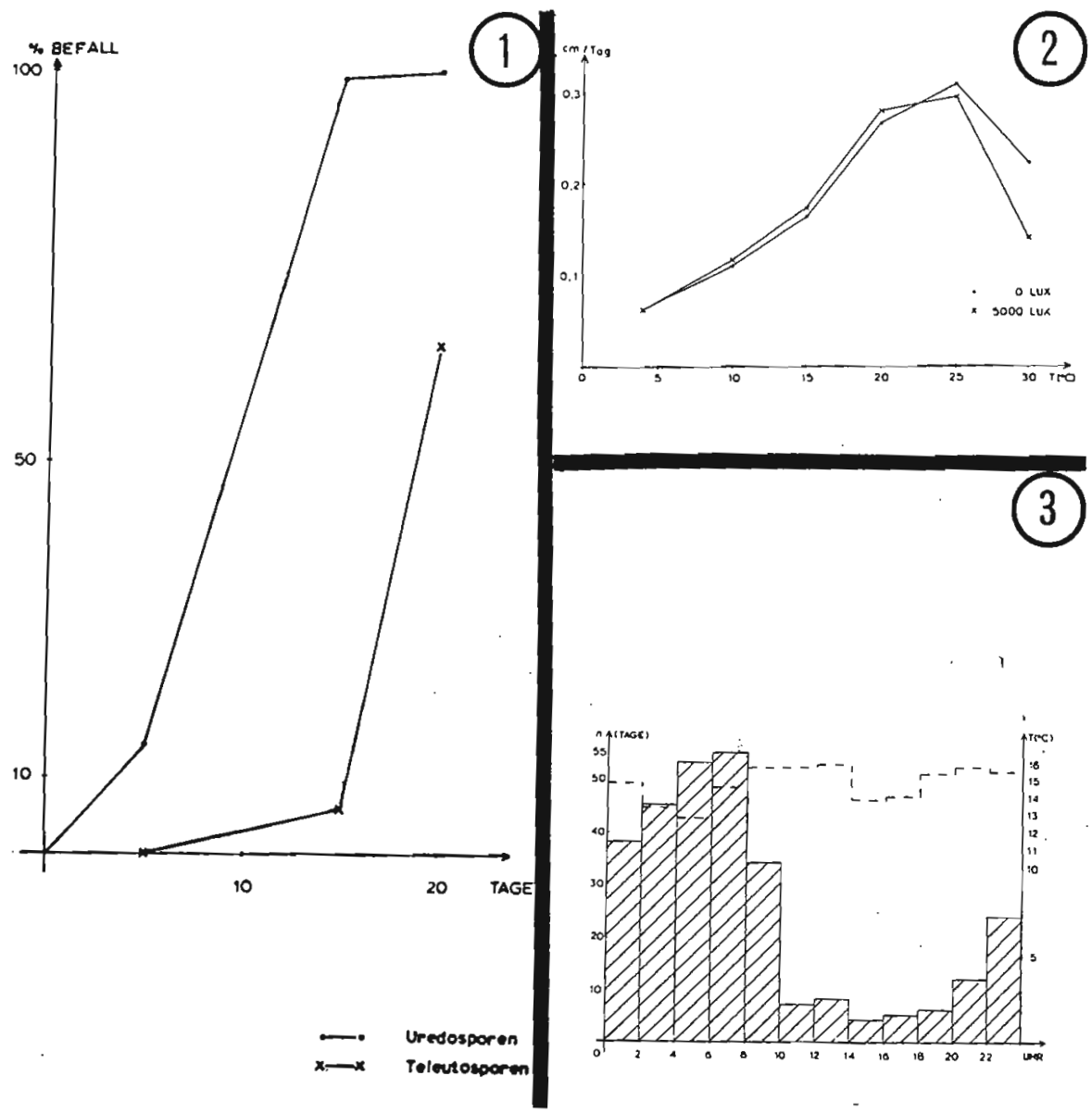

Abb. 1. Infektionsverlauf von $V$. lecanii auf Uredo- und Teleutosporen des Bohnenrostpilzes in Abhängigkeit von der Zeit. - Abb. 2. Myzelwachstum von $V$. lecanii auf Biomalzagar in Abhängigkeit von Temperatur und Licht. Wachstumsrate der Kolonien in $\mathrm{cm}$ pro Tag. Beleuchtung der Kulturen mit 10000 Lux. - Abb. 3. Auswertung der Klimadaten vom 22. Juni bis 22. August 1982. Dargestellt sind Tage, an denen rel. Luftfeuchtewerte über $90 \%$ auftraten. Aufgetragen wurde, zu welchen Tageszeiten und wie häufig Werte über $90 \%$ auftraten und welche mittleren Tagestemperaturen zu den entsprechenden Uhrzeiten vorlagen

Die Auswertung der Klimadaten vom 22. Juni bis zum 22. August 1982 (Abb. 3) ergab, daß am häufigsten rel. Luftfeuchtewerte über $90 \%$ erreicht wurden, wenn die Tagestemperaturen niedrig waren. Ein Vergleich mit Abb. 2 zeigt, daß bei einer Temperatur von $13,6^{\circ} \mathrm{C}$ (Durchschnittstemp. zwischen 2 Uhr und 8 Uhr) die Wachstumsrate des Hyperparasiten auf $53 \%$ reduziert ist. Obwohl rel. Luftfeuchten von über $90 \%$ pro Tag häufig 8 bis 12 Stunden lang vorkamen, reichte diese Zeitspanne nicht aus, um den Hyperparasiten im Feld zur Wirkung kommen zu lassen. 
Tabelle 2

Auswertung des Gewächshausversuches 1983

\begin{tabular}{|c|c|c|c|c|c|}
\hline Topf & GP/EPfl & $\mathrm{VP} / \mathrm{EPf}$ & Befall $\%$ & N/EPfl & G/EPAI \\
\hline R 1a & 134,94 & 2,41 & 1,79 & 7,23 & 30,54 \\
\hline R $1 b$ & 659,40 & 3,01 & 0,46 & 3,01 & 7,56 \\
\hline R $1 c$ & 661,39 & 0,00 & 0,00 & 5,70 & 14,53 \\
\hline$R 1 d$ & 324,67 & 0,00 & 0,00 & 5,33 & 10,47 \\
\hline R 2a & 576,80 & 3,20 & 0,55 & 3,20 & 9,50 \\
\hline$R 2 b$ & 976,76 & 2,82 & 0,29 & 3,52 & 3,61 \\
\hline $\mathrm{R} 2 \mathrm{c}$ & 949,33 & 0,67 & 0,07 & 4,00 & 11,44 \\
\hline $\mathrm{R} 2 \mathrm{~d}$ & 328,00 & 0,00 & 0,00 & 7,00 & 22,89 \\
\hline R 3a & 182,21 & 0,00 & 0,00 & 2,45 & 11,01 \\
\hline R $3 b$ & 367,31 & 1,44 & 0,39 & 2,40 & 7,32 \\
\hline $\mathrm{R} 3 \mathrm{c}$ & 432,48 & 0,00 & 0,00 & 3,42 & 16,02 \\
\hline R 3d & 678,70 & 0,00 & 0,00 & 6,48 & 19,62 \\
\hline R 4a & 257,89 & 0,00 & 0,00 & 3,76 & 5,14 \\
\hline $\mathrm{R} 4 \mathrm{~b}$ & 153,00 & 0,00 & 0,00 & 4,00 & 12,56 \\
\hline $\mathrm{R} 4 \mathrm{c}$ & 130,43 & 0,00 & 0,00 & 5,43 & 6,63 \\
\hline $\mathrm{R} 4 \mathrm{~d}$ & 290,00 & 2,67 & 0,92 & 5,33 & 15,27 \\
\hline $\mathrm{x}$ & 445,08 & 1,01 & 0,28 & 4,52 & 12,76 \\
\hline$s$ & 275,77 & 1,32 & 0,49 & 1,57 & 7,04 \\
\hline V 1a & 292,00 & 50,00 & 17,12 & 7,00 & 17,35 \\
\hline $\mathrm{V} 1 \mathrm{~b}$ & 109,00 & 0,00 & 0,00 & 7,00 & 7,87 \\
\hline V 1c & 187,18 & 11,11 & 5,94 & 5,98 & 16,74 \\
\hline V 1d & 176,06 & 19,01 & 10,80 & 4,93 & 13,69 \\
\hline $\mathrm{V} 2 \mathrm{a}$ & 34,94 & 0,00 & 0,00 & 4,82 & 22,46 \\
\hline $\mathrm{V} 2 \mathrm{~b}$ & 141,30 & 7,61 & 5,38 & 7,61 & 14,12 \\
\hline $\mathrm{V} 2 \mathrm{c}$ & 200,93 & 33,33 & 16,59 & 3,70 & 12,06 \\
\hline $\mathrm{V} 2 \mathrm{~d}$ & 175,35 & 7,04 & 4,02 & 4,22 & 7,96 \\
\hline V 3a & 24,10 & 0,00 & 0,00 & 7,23 & 26,33 \\
\hline V $3 b$ & 43,52 & 0,00 & 0,00 & 5,56 & 21,63 \\
\hline $\mathrm{V} 3 \mathrm{c}$ & 110,76 & 21,52 & 19,43 & 3,80 & 12,35 \\
\hline V 3d & 59,78 & 11,96 & 20,00 & 11,96 & 20,43 \\
\hline V 4a & 13,33 & 0,00 & 0,00 & 10,67 & 26,20 \\
\hline V 4b & 583,76 & 21,37 & 3,66 & 7,69 & 17,38 \\
\hline $\mathrm{V} 4 \mathrm{c}$ & 106,29 & 8,57 & 8,06 & 3,43 & 10,73 \\
\hline $\mathrm{V} 4 \mathrm{~d}$ & 12,04 & 0,93 & 7,69 & 3,70 & 6,02 \\
\hline $\mathrm{x}$ & 141,90 & 12,03 & 7,42 & 6,21 & 15,83 \\
\hline$s$ & 142,37 & 14,20 & 7,30 & 2,50 & 6,34 \\
\hline
\end{tabular}

R: $\quad$ Nur mit Bohnenrost behandelter Versuchsteil

V: $\quad$ Mit V. lecanii behandelter Versuchsteil

EPfl.: Die Blätter pro Pflanze wurden standardisiert, 1 EPfl. hat 4 Blätter

GP/EPfl.: Gesamtrostpusteln pro Einheitspflanze

VP/EPfl.: Mit V. lecanii befallene Rostpusteln pro Einheitspflanze

Befall: Rostpusteln mit $V$. lecanii befallen in \%

N/EPfl.: Anzahl geemteter Bohnen pro Einheitsplanze

G/EPfl: : Gewicht der Bohnen pro Einheitspflanze

$\mathrm{x}$ : Mittelwert

s: $\quad$ Standardabweichung 


\section{Biologische Bekämpfung im Gewächshaus}

Ausgewertet wurde die Anzahl Rostpusteln auf den Blättern der, die Versuchspflanzen flankierenden, unbehandelten Bohnenpflanzen (Tab. 1). Die Anzahl Blätter/Pflanze wurde standardisiert. Die Ausbreitung des Bohnenrostpilzes auf benachbarte Pflanzen konnte mit einer Konfidenz von 99,0 \% zu 68,12\% verhindert werden. Für einen Mehrertrag in Stück Bohnen/Pflanze von 27,68\% gab es nur $95 \%$ Konfidenz, für einen Mehrertrag in kg Bohnen/Pflanze ergaben sich keine signifikanten Werte (Tab. 2). Weiterhin wurde die Ausbreitung des Hyperparasiten auf mit Bohnenrost infizierten Pflanzen im Versuchsteil "R ${ }^{“}$ nachgewiesen. Hierfür wurden mit $V$. lecanii infizierte Trauermücken der Gattung Sciara spec. (HECK, mündliche Mitteilung) verantwortlich gemacht, die zur Verbreitung des Pilzes beitrugen. Tote, von Myzel des Hyperparasiten überwucherte Insekten wurden regelmäßig gefunden.

\section{Diskussion}

Hyperparasiten gegen pflanzenparasitierende Pilze, wie zum Beispiel die biotrophen Rostpilze, einzusetzen und sie somit für den Pflanzenschutz nutzbar zu machen, ist ein Ziel, dem heute große Bedeutung zugemessen wird. Hier bietet sich die Möglichkeit, die Schadpilze in ihrem Vermehrungszyklus an Punkten zu treffen, wo jedes chemische Bekämpfungsmittel versagt: Inaktivierung und Abbau von Uredosporen (Vermehrungspotential) und von Teleutosporen (Überwinterungspotential). Die Untersuchung von Einfluß der Temperatur und der Lichtverhältnisse auf das Wachstum des Hyperparasiten ergab eine sehr gute Übereinstimmung mit Еквом (Еквом und AFMAN 1980). Zwischen $4{ }^{\circ} \mathrm{C}$ und $15^{\circ} \mathrm{C}$ ist eine fast lineare Zunahme der Wachstumsrate festzustellen, bei $25^{\circ} \mathrm{C}$ ist ein Optimum erreicht und danach nimmt die Wachstumsgeschwindigkeit/Tag rapide $a b$. In dieser Phase wurde zum ersten $\mathrm{Mal}$ ein signifikanter Einfluß von Licht erkennbar. Beleuchtung scheint bei Temperaturen über $30^{\circ} \mathrm{C}$ nachteilig auf den Metabolismus von $V$. lecanii zu wirken. Auf welche Ursachen dieser Einfluß zurückzuführen ist, konnte nicht geprüft werden.

Die Zeitspanne von 15 Tagen bis zur $100 \%$ igen Infektion der Uredosporen erhöht die Wahrscheinlichkeit einer Rostsporenverbreitung, bevor die Aktivität des Hyperparasiten wirksam werden kann. Die starke Verzögerung der Infektion von Teleutosporen durch $V$. lecanii, nach 15 Tagen erst knapp $6 \%$, wird auf die verstärkte und mit Melanin durchsetzte Sporenwand der Überwinterungssporen zurückgeführt, die eine Penetration des Hyperparasiten erschwert, nicht jedoch aufhält (BLOOMFIELD und ALEXANDER 1967).

Eine Bekämpfung von $U$. appendiculatus var. appendiculatus im Freiland war nicht möglich. Die Seenähe des Versuchsgartens gewährleistete ein häufiges Auftreten von rel. Lufffeuchten über $90 \%$. Prozentual in bezug auf die Gesamtversuchsdauer gesehen, hielten die hohen Luftfeuchtewerte zu 48,3\% zwischen 8 und 12 Stunden/Tag an. Alleine durch den Faktor Luftfeuchte kann das schlechte Wachstum des Hyperparasiten nicht erklärt werden. In den frühen Morgenstunden wurden rel. Lufffeuchtewerte über $90 \%$ am häufigsten gemessen, überwie- 
gend zusammen mit niedrigen Temperaturen (Abb. 3). Bei einem Schnitt um $13,6{ }^{\circ} \mathrm{C}$ ist die Wachstumsrate des Hyperparasiten um ca. die Hälfte vermindert. Außerdem kann angenommen werden, daß im Hochsommer Temperaturen von über $25^{\circ} \mathrm{C}$ unter Bestrahlung erreicht werden. Sollte der Einfluß von Licht bei hohen Temperaturen zu einer direkten oder indirekten Schädigung des Myzels von $V$. lecanii führen, so muß hier ein möglicher, nicht gemessener, negativer Faktor zusätzlich in Betracht gezogen werden (der Thermohygrograph war beschattet).

In der Schlußfolgerung muß darauf hingewiesen werden, daß weitere Untersuchungen notwendig sind, bei denen alle Faktoren direkt am Blatt gemessen und zusätzliche Maßnahmen zur Unterstützung des Wachstums des Hyperparasiten durch eine geeignete Formulierung des Sporenmaterials getroffen werden sollten. Versuche im Gewächshaus fanden bei rel. Lufffeuchtewerten um $90 \%$ statt. Der Bekämpfungserfolg der Rostausbreitung betrug $68 \%$, im Vergleich zu der nicht behandelten Kontrollparzelle. In den umliegenden Gewächshäusern in Wahlwies und auf der Reichenau hatte $V$. lecanii keinen Erfolg in der Schädlingsbekämpfung (HECK, persönliche Mitteilung). Dies muß auf die Bewässerungsmethode in den Häusern zurückgeführt werden. Für den Einsatz von Hyperparasiten, speziell Raubmilben (HECK, persönliche Mitteilung) und hyperparasitäre Pilze wie Ampelomyces quisqualis (PHILIPP 1979) und V. lecanii ist die Tröpfchenbewässerung wegen der ariden Klimabedingungen, die mit ihr verbunden sind, unakzeptabel. Vielleicht wird dem Landwirt die Entscheidung zugunsten des "Integrierten Pflanzenschutzes“ erleichtert, wenn die Effizienz der Hyperparasiten durch geeignete Formulierungen weiter gesteigert werden kann und Pestizide verfügbar sind, die mit dem Einsatz der Hyperparasiten vereinbar sind. Untersuchungen dazu sind für $V$. lecanii vorgelegt worden (WILDING 1972).

\section{Literaturverzeichnis}

BloOMfield, B. J., and M. AleXander, 1967: Melanins and resistance of fungi to lysis. J. Bacteriol. 93, $1276-1280$.

DeAcon, J. W., 1983: Microbial control of plant pests and diseases. 4.: Microbial control of pests, use of fungi. In: DeAcon, J. W. (Edit.). Aspects of Microbiology, Nr. 7 Van Nostrand Reinhold (UK) Co. Ltd., Wokingham, England.

Domsch, K. H., 1960: Das Pilzspektrum einer Bodenprobe III. Nachweis der Einzelpilze. Arch. Mikrobiol. 35, 310-339.

Еквом, B. S., and J. AhмаN, 1980: The fungus Verticillium fusisporum as an insect pathogen. J. Invertebr. Pathol. 36, 136-138.

Flanagan, P. W., and A. M. Scarborough, 1974: Physiological groups of decomposer fungi on tundra plant remains. In: HolDING, A. J. (Edit.). Soil organisms and decomposition in tundra. Biome Steering Comitee, Stockholm, 159-181.

GAMs, W., 1971: Cephalosporium-artige Schimmelpilze (Hyphomycetes). Fischer Verlag, Stuttgart.

HÄNSSLER, G., M. KNÖRZER, and H. J. REISENER, 1981: Lichmikroskopische Untersuchungen der Interaktion zwischen Puccinia graminis var. tritici und Verticillium lecanii. Phytopath. Z. 102, 310-319.

- -, M. HermanNs, und H. J. Retsener, 1982: Elektronenmikroskopische Beobachtung der Interaktion zwischen Uredosporen von Puccinia graminis var. tritici und Verticillium lecanii. Pbyropath. Z. 103, 139-148. 
Hans, R., 1983: Gesundheitliche Aspekte biologischer Pflanzenschutzmittel. Deurscher Gartenbau 21, 982-983.

Hassebrauk, K., 1936: Pilzliche Parasiten der Getreideroste I. Phytopath. Z. 9, 513-516.

- -, 1937: Pilzliche Parasiten der Getreideroste II. Phytopath. Z. 10, 465.

HeRING, T. F., 1965: Oakwood litter fungi. Trans. Br. Mycol. Soc. 48, 391-408.

MENDGEN, K., 1979 a: Können Hyperparasiten Fungizide ersetzen? Arbeitsber. ökol. Umwelttechn., Univ. Konstanz 3, 81-87.

_- 1979 b: Verticillium lecanii, ein Hyperparasit auf dem Getreidegelbrost Puccinia striiformis. Mitt. Biol. BundAnst. Land- und Forstw., Berlin, 191, 301-302.

- 1981: Growth of Verticillium lecanii in pustules of stripe rust Puccinia graminis. Phytopath. Z. 102, 301-309.

PhLrP, W.-D., and G. CRUger, 1979: Parasitismus von Ampelomyces quisqualis auf Echten Mehltaupilzen an Gurken und anderen Gemüsearten. Z. Pflanzenkr. und Pflanzenschutz 86, $129-142$.

- -, 1983: Schadpilze im Gartenbau biologisch bekämpfen. Deutscher Gartenbau 21, 978 980.

SCHROEDER, H. voN, und K. Hassebrauk, 1957: Beiträge zur Biologie von Darluca filum (Bio.) und einigen anderen, auf Uredineen beobachteten Pilzen. Zentralbl. Bakt., II. Abt., 110, $676-696$.

WIIDING, N., 1972: The effect of systemic fungicides on the aphid pathogen Cephalosporium aphidicola. Plant Physiol. 21, 137-139.

Adresse der Autoren: Dr. Christian Grabsru, Sandoz AG, Agrobiologische Versuchsstation, CH-4107 Witterswil (Schweiz). Prof. Dr. KuRt MENDGen, Fakultät für Biologie, Lehrstuhl,Phytopathologie, Universität Konstanz, D-7750 Kostanz (F,R.G.). 